\title{
APERTURA Y PRODUCTIVIDAD TOTAL DE LOS FACTORES: ANALISIS DE LA CONTEMPORANEIDAD EN LOS QUIEBRES ESTRUCTURALES PARA AMERICA LATINA Y EL CARIBE*
}

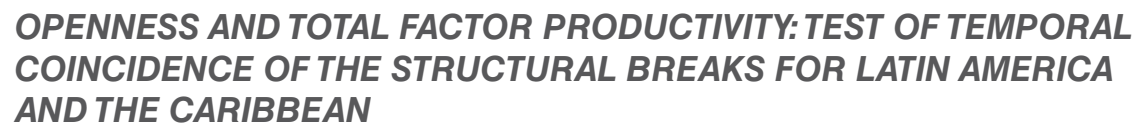

\section{GERMAN HECTOR GONZALEZ** \\ CONICET y Universidad Nacional del Sur (Argentina)}

\section{FERNANDO ANDRES DELBIANCO***}

Universidad Nacional del Sur y Universidad Torcuato di Tella (Argentina)

\begin{abstract}
We study the existence of a structural break in TFP and in several indicators of openness for a sample of 20 Latin American and Caribbean economies for the period 1960-2005. For this purpose, the tests of Zivot and Andrews (1992) and Bai and Perron (1998) on a series of TFP computed from a development accounting exercise (Hsieh and Klenow, 2010) and alternative measures of openness with different characters (Wacziarg, 2001) have been used. The cases of breaks on TFP during the consolidation process of openness in the region (1985-1995) are not significant. Shocks in openness seem to have effects on the rate of growth of TFP.
\end{abstract}

Keywords: Openness, Total Factor Productivity, Latin America and the Caribbean.

JEL Classification: F4, O38, O47, N16.

\section{Resumen}

Se ha estudiado la existencia de quiebres estructurales en la PTF y en varios indicadores de apertura para una muestra de 20 economías latinoamerica-

\footnotetext{
* Los autores desean agradecer los comentarios del árbitro de la revista y los recibidos por Gabriel Michelena y los asistentes a la presentación que se hizo de una versión anterior durante la XLV Reunión Anual de la Asociación Argentina de Economía Política. Los errores y omisiones son nuestros.

** ghgonza@uns.edu.ar

*** fernando.delbianco@alumni.utdt.edu
} 
nas y del Caribe para el período 1960-2005. Se han utilizado los tests de Zivot y Andrews (1992) y el de Bai Perron (1998) sobre una serie de PTF computada a partir de un ejercicio de contabilidad del desarrollo (Hsieh y Klenow, 2010) y sobre medidas alternativas de apertura con diferentes caracteres (Wacziarg, 2001). Los casos de quiebre en la PTF durante el proceso de consolidación de la apertura en la región (1985-1995) no son significativos. Los resultados parecen indicar que los shocks en la apertura operan sobre las tasas de crecimiento de la PTF.

Palabras Clave: Apertura, Productividad Total de los Factores, America Latina y el Caribe.

Clasificación JEL: F4, O38, O47, N16.

\section{INTRODUCCION}

La literatura aún discute sobre la existencia o no de una relación causal teórica, de largo plazo, entre apertura al comercio internacional y productividad. Hipótesis que ha sido tantas veces verificada como rechazada. Sin embargo, se podría afirmar que actualmente se habría llegado a cierto acuerdo en que la apertura tiene, al menos, la capacidad de motivar decisiones que repercuten en la productividad total de los factores (PTF) en forma de mejoras tecnológicas en bienes y en procesos de producción, aunque el resultado concreto depende de otros factores no siempre controlables (Baldwin, 2003; Rodrik, 2003; Kneller et al., 2008).

Nehru y Dhareshwar (1993) tempranamente llegaron a esa conclusión. Contrariamente, Edwards (1998) sostuvo que existe una relación positiva y robusta entre apertura y la tasa de crecimiento en la PTF, aunque la robustez fue puesta en duda por Rodríguez \& Rodrik (2000). Los resultados presentados en el artículo de Benjamin y Ferrantino (2001) no son concluyentes según sus mismos autores. Mientras que Haouas y Yagoubi (2005) encuentran que una mayor apertura causa tasas mayores de crecimiento de la PTF en las economías del MENA (Middle East and North Africa), y Söderbom y Teal (2003) realizan exactamente el mismo ejercicio para todas las regiones y niveles de ingreso encontrando el mismo resultado. En cambio, González y Constantin (2009) sostienen que esa relación no es significativa en economías de ingresos bajos, aunque sí lo es en aquellas de ingresos medio y alto. Das (2002), luego de examinar 37 artículos que estudian la relación entre apertura y la tasa de crecimiento de la productividad industrial, llega a la conclusión que existiría una positiva relación entre ambas variables en América Latina, Asia y África, aunque señala que hay que interpretar los resultados con cautela porque el "análisis empírico está plagado de limitaciones" (p. 36). Tomando la PTF en niveles, en vez de tasas de crecimiento, Miller y Upadhyay (2000 y 2002), y González (2002) encuentran que una mayor exposición al comercio internacional es en general positiva para la PTF, pero no es robusta esta relación cuando se dividen los países en grupos y se introducen variables adicionales. Por su parte, Alcalá y 
Ciccone (2004) encuentran que el efecto positivo es significativo y estadísticamente robusto. Herzer (2005) y Jonsson y Subramanian (2001) utilizan técnicas de series de tiempo encontrando que la relación existe y es significativa para el caso de Chile y Sudáfrica, respectivamente. Calvanti Ferreira y Trejos (2006) muestran que las restricciones al comercio explican una importante proporción de la brecha en la PTF, fundamentalmente para las economías de ingresos medios.

Cualquiera sea el resultado de esta discusión, los hechos indican que las economías en general han mostrado en los últimos dos siglos, y en particular en los últimos 60 años, una creciente interdependencia. Esto ha sido como resultado en muchos casos de políticas que explícitamente tuvieron como objetivo lograr el desarrollo económico a partir de la apertura de la economía doméstica al comercio internacional. El timing de dicha apertura y sus resultados varían según el caso. El objetivo de este artículo es diferente a la literatura precedente. Se pretende observar si existe contemporaneidad entre los cambios abruptos en la apertura al comercio internacional y los cambios sustanciales en la productividad, es decir, si los primeros son reproducidos en forma de cambios significativos en el sendero de largo plazo de la productividad total de los factores de 20 economías latinoamericanas y caribeñas entre 1960 y 2005. Se considera contemporáneos a los quiebres estructurales de al menos dos series si el quiebre en una de ellas coincide o se encuentra dentro de los dos años de transcurrido el quiebre en la restante.

El estudio del timing de la apertura es relevante en el siguiente sentido. Un quiebre en la política comercial (independientemente del sentido que tenga) acarrea la destrucción de capacidad instalada asociada al ajuste inmediato de la estructura productiva. Suponiendo que es posible observar una estrecha relación positiva en el largo plazo entre apertura y productividad, y los cambios en la primera se reproducen inmediatamente en la segunda, entonces la apertura unilateral y de una vez sería una política recomendable, siempre que el ambiente político y económico fuera el apropiado, porque los beneficios de la ganancia de productividad permitirían compensar los costos sociales del ajuste. En cambio, si los cambios en la productividad son graduales, los beneficios de la apertura pueden verse diluidos en el tiempo y un cambio escalonado hacia la liberalización del comercio internacional podría ser la opción recomendable. En este último caso, una política exterior que profundice y amplíe los acuerdos de integración regional podría ser más apropiada, en lugar de una liberalización unilateral.

Se utilizan los tests de Zivot y Andrews (1992) y de Bai y Perron (1998) para determinar si las economías han sufrido, y cuándo, cambios significativos en las medidas de apertura y productividad total de los factores, esta última tomada en niveles y en tasas de crecimiento. Luego se comparan los puntos de quiebre de las series para analizar si existe contemporaneidad. Finalmente, se analiza la contemporaneidad con otros hechos relevantes durante el período en consideración que pudieron haber afectado significativamente a la PTF. La estimación de la PTF se realiza a partir del residuo de la función agregada de producción siguiendo la metodología propuesta por la contabilidad del desarrollo (Hsieh y Klenow, 2010). Para reducir la discrecionalidad en la medición de la apertura se toman cuatro medidas alternativas, que corresponden a medidas de resultado, de política y de desviación, también se utiliza la literatura 
especializada sobre el proceso de liberalización del comercio en América Latina y el Caribe para generar una periodización que se contrastará con las estimaciones. Finalmente se utilizan otros hechos de la historia económica reciente para encuadrar los shocks estimados en la PTF.

El resultado más importante que se encuentra es que luego de un proceso abrupto de apertura (o cierre), los cambios en la PTF, de existir, son graduales. Al menos en la región, los cambios abruptos en la PTF estuvieron, en general, asociados a factores de otra índole (v. gr. Crisis del Petróleo). Por otra parte, los casos de quiebre de la PTF, en niveles, durante el proceso de consolidación de la apertura en la región (1985-1995) no son significativos. Mientras que en tasas de crecimiento de la PTF se observa una mayor cantidad de casos de contemporaneidad, aunque no existirían diferencias en la frecuencia entre el período de consolidación de la apertura y el período inmediato anterior (1973-1984). Tampoco existe contemporaneidad entre apertura, niveles y tasas de crecimiento de la PTF.

La siguiente sección describe la metodología empleada. La tercera sección muestra los resultados obtenidos a partir de la aplicación de los test de corte estructural y se analiza la contemporaneidad. La cuarta sección concluye.

\section{METODOLOGIA Y DATOS}

\subsection{Detección de posibles quiebres estructurales}

Para este trabajo se realiza en primera instancia un test de raíz unitaria como el Zivot y Andrews (1992) que sugiere un quiebre estructural bajo la hipótesis alternativa. Para robustecer los resultados se implementa el test de quiebre estructural de Bai y Perron (1998-2003) para cambios estructurales puros. Es decir, que se estudian las series uniecuacionalmente sin sugerir necesariamente un modelo subyacente que explique el comportamiento de cada una de ellas (tanto de la productividad de los factores como de los indicadores de apertura seleccionados para el estudio de la contemporaneidad).

El clásico test de Dickey-Fuller aumentado (ADF) con tres especificaciones distintas (con tendencia, con intercepto, y sin constante) para testear estacionariedad sin quiebre estructural en las series utilizadas arroja que, en general, las series no son estacionarias. Sin embargo, estos resultados no sorprenden cuando se trabaja con largas series de tiempo. En el anexo se muestran estos resultados.

Los test de raíz unitaria comunes, como el conocido Dickey-Füller (Dickey y Füller, 1984) o el test de Perron (Perron, 1989), tienden a no rechazar la hipótesis nula de raíz unitaria en presencia de cambios estructurales, y por ende concluir que se está en presencia de series no estacionarias. Existen test que permiten aclarar dónde hay un cambio estructural, como por ejemplo el test de Chow, sin embargo se debe contar con información previa sobre la existencia de un posible punto de quiebre o recurrir a la iteración para encontrarlos. Diferente es encontrar endógenamente el mencionado cambio, para lo cual se recurre al test que Zivot y Andrews crearon en 1992. 
El test de Zivot y Andrews (ZA) analiza la posible presencia de cambio estructural de la serie en cada una de las observaciones, de manera secuencial, generando variables dummy en cada período. Luego, aquella variable dummy con mayor significatividad es tomada como el punto en el que la serie sufre un cambio estructural. Aunque no deja de ser un test de raíz unitaria, se diferencia con los anteriores mencionados al tener en cuenta la posible existencia de quiebres estructurales.

Se buscan tres posibles especificaciones: El modelo I se encuentra especificado para buscar un cambio de intercepto, y se busca el máximo rezago de la serie mediante el test de la t; el modelo II es similar al anterior, pero permitiendo sólo cambios tendencia; finalmente, el modelo III evalúa la posibilidad de cambios tanto en intercepto como en tendencia, y utiliza el test de AIC para determinar el máximo número de rezagos de la serie. Para los tres posibles quiebres especificados se trabaja en un nivel de significatividad del $5 \%$.

Modelo I: Modelo con intercepto

$$
\Delta y_{t}=\gamma+\alpha y_{t-1}+\beta t+\psi D I_{t}+\sum_{j=1}^{k} d_{j} \Delta y_{t-j}+\mu_{t}
$$

Modelo II: Modelo con tendencia

$$
\Delta y_{t}=\gamma+\alpha y_{t-1}+\beta t+\lambda D T_{t}+\sum_{j=1}^{k} d_{j} \Delta y_{t-j}+\mu_{t}
$$

Modelo III: Modelo con ambas especificaciones

$$
\Delta y_{t}=\gamma+\alpha y_{t-1}+\beta t+\psi D I_{t}+\lambda D T_{t}+\sum_{j=1}^{k} d_{j} \Delta y_{t-j}+\mu_{t}
$$

donde $\gamma$ es el intercepto, $\beta t$ es la tendencia, $\sum_{j=1}^{k} d_{j} \Delta y_{t-j}$ son los rezagos que se espe-
cifiquen y $\mu_{t}$ es el error aleatorio.

Como se puede observar, la especificación no dista mucho del test de DickeyFüller, sólo que añade las dummies $\lambda D T_{t}$, y $\psi D I_{t}$ para capturar un posible quiebre estructural, permitiendo cambio en la tendencia o en el intercepto respectivamente, y detectarlo endógenamente.

La hipótesis nula es, en los tres modelos, que $\alpha=0$, es decir, que existe estacionariedad y no hay ningún quiebre estructural, mientras que la hipótesis alternativa descarta la estacionariedad y propone un quiebre estructural en algún punto de la serie temporal.

Principalmente, el foco de atención se pone en el tercer modelo que deja abierta la posibilidad de la existencia tanto de quiebre en intercepto como en tendencia. La razón es que si se especifica el modelo I o el modelo II, cuando el modelo real es distinto al especificado, se pierde mucha potencia en el test. En cambio, si se especifica el modelo III, pero el verdadero modelo subyacente es el uno o el dos, la pérdida de potencia es menor (Sen, 2003). 
Para completar el estudio de las series de tiempo se contrastan los resultados a partir de Zivot y Andrews, con los obtenidos a partir del test de quiebre estructural sugerido por Bai y Perron (1998). Esta metodología considera el siguiente modelo múltiple con $m$ número de quiebres estructurales:

$$
\begin{array}{ll}
y_{t}=x_{t}^{\prime} \beta+z_{t}^{\prime} \delta_{1}+\mu_{t}, & t=1, \ldots, T_{1} \\
y_{t}=x_{t}^{\prime} \beta+z_{t}^{\prime} \delta_{2}+\mu_{t}, & t=1, \ldots, T_{2} \\
\cdots & \\
y_{t}=x_{t}^{\prime} \beta+z_{t}^{\prime} \delta_{m+1}+\mu_{t}, & t=T_{m}+1, \ldots, T
\end{array}
$$

donde $y_{t}$ es la variable dependiente observada en el tiempo $t, x_{t}$ es la matriz de coeficientes de la regresión, $\beta$ y $\delta_{j}(j=1, \ldots, m+1)$ son los respectivos vectores de coeficientes y $\mu_{t}$ es el término de error en el tiempo.

Los puntos de quiebre $\left(T_{1}, \ldots, T_{m}\right)$ son tratados como desconocidos y son estimados junto con los coeficientes de las $T$ observaciones disponibles. La hipótesis nula de este test es la no existencia de quiebres contra la alternativa de un desconocido número de quiebres. Suponiendo un quiebre estructural puro como hemos mencionado anteriormente, es decir que toda la estructura cambia en conjunto, tenemos que:

$$
Y=\bar{Z} \delta+U
$$

donde $\bar{Z}$ es una matriz diagonal en bloques, donde cada bloque corresponde a uno de los $m$ regímenes especificados $\left(T_{1}, \ldots, T_{m}\right)$ y $\delta=\left(\delta_{1}^{\prime}, \ldots, \delta_{m+1}^{\prime}\right)$ son los coeficientes que acompañan a los quiebres.

Los quiebres hallados son aquellos que cumplen la siguiente condición:

$$
\left(\hat{T}_{1}, \ldots, \hat{T}_{m}\right)=\arg \min _{T_{1}, \ldots, T_{m}} S_{T}\left(T_{1}, \ldots . T_{m}\right)
$$

donde $S_{T}\left(T_{1}, \ldots T_{m}\right)$ es la suma total de residuos al cuadrado resultante de la $m$ partición elegida.

\subsection{Estimación de la PTF}

El punto de partida es una función de tipo Cobb-Douglas con tres factores productivos y una variable que captura el nivel de productividad multifactorial. Esta última se computa como el residuo en la función de producción. Se supone que todas las economías de la muestra pueden ser explicadas mediante el mismo modelo; por consiguiente, las diferencias estructurales entre ellas que pudieran existir corresponden al residuo de esta función agregada. 
Se representa al producto, $Y$, mediante la siguiente expresión:

$$
Y_{i}=K_{i}^{\alpha} H_{i}^{\beta}\left(\mathrm{A}_{i} L_{i}\right)^{1-\alpha-\beta}
$$

donde $i$ denota la economía, $\alpha$ y $\beta$ son las participaciones del capital físico, $K$, y humano, $H$, en el producto, respectivamente $(\alpha+\beta<1)$. El stock de capital humano es el producto entre el nivel medio de capital humano, $h$, y el número de trabajadores, $L\left(H_{i}=h_{i} \times L_{i}\right)$. La variable A representa el nivel de productividad multifactorial o productividad total de los factores. A su vez, se supone que todas las economías poseen los mismos valores de los parámetros.

Se busca alcanzar una expresión que relacione al ingreso per cápita con las intensidades factoriales y la productividad multifactorial. Denotando con $P_{i}$ a la población de la economía $i$, la expresión (2) expone al ingreso per cápita y su composición:

$$
\frac{Y_{i}}{P_{i}}=\frac{L_{i}}{P_{i}}\left(\frac{K_{i}}{Y_{i}}\right)^{\frac{\alpha}{1-\alpha-\beta}}\left(\frac{H_{i}}{Y_{i}}\right)^{\frac{\beta}{1-\alpha-\beta}} \mathrm{A}_{i}
$$

Los tres primeros componentes de la derecha brindan una aproximación de la relevancia que posee la acumulación de factores en el producto per cápita. La variable $L / P$ es la tasa de ocupación y captura el efecto que, sobre el ingreso per cápita, tiene la cantidad del factor trabajo empleado en el proceso productivo. Las variables $K / Y$ y $H / Y$ son la intensidad del capital físico y humano en el producto. $A$ es la productividad multifactorial o PTF, y posee dos grandes fuentes de variación. La primera, llamada "capacidades tecnológicas", representa "un conjunto complejo de habilidades humanas, conocimiento tecnológico, estructura organizacional, requeridos para operar eficientemente tecnología y alcanzar un proceso de cambio tecnológico" (Llal, 1992). Es decir, que no sólo implica eficiencia productiva sino la capacidad para adaptar y mejorar la tecnología disponible a las posibilidades reales de cada economía. La segunda fuente captura los saltos en la frontera de posibilidades de producción, esto es, cambios en la tecnología como resultado del proceso endógeno de aprendizaje o incorporados desde el exterior (a través de importaciones de bienes y/o patentes, inversión externa, inmigración, etc.), lo suficientemente importantes como para alterar considerablemente los “costos reales de producción” (Harberger, 1998).

Finalmente,

$$
\mathrm{A}_{i}=\frac{\frac{Y_{i}}{P_{i}}}{\frac{L_{i}}{P_{i}}\left(\frac{K_{i}}{Y_{i}}\right)^{\frac{\alpha}{1-\alpha-\beta}}\left(\frac{H_{i}}{Y_{i}}\right)^{\frac{\beta}{1-\alpha-\beta}}}
$$


Para la calibración de (3) se supone $\alpha=0.31$ y $\beta=0.28$ siguiendo a Mankiw et al. (1992), Klenow y Rodríguez-Clare (1997), McGrattan y Schmitz (1999), Hopenhayn y Neumeyer (2004). Esta misma metodología y valores de los parámetros se utilizó en González et al. (2009) y se encontró que no existen, para los datos utilizados en el artículo, diferencias sustanciales en las conclusiones ante cambios en los valores de los parámetros. Las fuentes de datos y los procedimientos de cómputo se detallan en el anexo.

El panel de datos está constituido con información de veinte economías americanas para un período de 46 años (1960-2005). Su composición es diez economías sudamericanas, siete de América Central y del Norte, y tres del Caribe. Específicamente, Argentina, Bolivia, Brasil, Chile, Colombia, Costa Rica, Ecuador, El Salvador, Guatemala, Honduras, Jamaica, México, Nicaragua, Panamá, Paraguay, Perú, República Dominicana, Trinidad y Tobago, Uruguay, Venezuela. De acuerdo a la clasificación del Banco Mundial según nivel de ingreso correspondiente al ejercicio 2005, son doce economías de ingreso medio bajo y ocho de ingreso medio alto.

\subsection{Medidas de apertura}

Ante los problemas que presentan los diferentes indicadores de apertura (Wacziarg, 2001; Pritchett, 1996; Anderson y Neary, 1992; Krishna, 1991; entre otros) se tomaron tres tipos de indicadores:

a) una medida de resultado en dos versiones: la intensidad comercial, computada de la forma tradicional como el cociente entre el total del comercio (exportaciones e importaciones) y el producto bruto interno. Una primera versión tomando las variables en dólares a precios constantes, $(\mathrm{X}+\mathrm{M}) / \mathrm{PIB}$, y una segunda versión tomando las variables en dólares corrientes $(\mathrm{X}+\mathrm{M}) / \mathrm{PIB} *$.

b) un indicador de política: siendo $\mathrm{r}$ la tasa promedio de impuestos sobre las transacciones comerciales, se define la tasa de apertura como 1-r. La tasa $r$ se computa como el cociente entre la recaudación total tributaria por gravámenes a las transacciones internacionales (exportaciones e importaciones) y el total del comercio internacional (exportaciones más importaciones).

c) un indicador de desviación: corresponde a la desviación de la intensidad comercial respecto a la predicha teniendo en cuenta las características estructurales de la economía en particular y el resto de la muestra, u. Las características que se tienen en cuenta son el tamaño de su población, superficie, producto per cápita, si son exportadores de petróleo y si son islas.

\section{RESULTADOS}

Las gráficas de todas las series utilizadas para cada economía se encuentran en el anexo. Para la PTF tomada en niveles, el test ZA encuentra potenciales puntos de quiebre estructural en las veinte economías, sin embargo al 5 y $1 \%$ de significatividad solo en una y cinco oportunidades, respectivamente. En el caso de la apertura medida por el indicador de intensidad comercial, $(\mathrm{X}+\mathrm{M}) / \mathrm{PIB}$, el test ZA encuentra potenciales puntos de quiebre estructural en todas las economías, sin embargo significativa al $5 \%$ 
solo en dos oportunidades. En cuanto al indicador de política comercial, 1-r, sobre 16 economías de las que se cuentan suficiente información, se encontraron casos significativos de corte estructural en nueve oportunidades, siete de los cuales al $1 \%$. Los resultados se muestran en la Tabla 1.

\section{TABLA 1}

QUIEBRES ESTRUCTURALES EN LAS SERIES DE PTF Y DOS INDICADORES DE APERTURA SEGUN EL TEST DE ZA

\begin{tabular}{|c|c|c|c|c|c|c|c|c|c|}
\hline \multirow[b]{2}{*}{ País } & \multicolumn{3}{|c|}{ PTF } & \multicolumn{3}{|c|}{$(\mathrm{X}+\mathrm{M}) / \mathrm{PIB}$} & \multicolumn{3}{|c|}{$1-\mathrm{r}$} \\
\hline & Quiebre & Modelo & Signif. & Quiebre & Modelo & Signif. & Quiebre & Modelo & Signif. \\
\hline Argentina & 1981 & II & n.s. & 1982 & II & n.s. & 1991 & II & n.s. \\
\hline Bolivia & 1979 & II & n.s. & 1980 & II & n.s. & 1988 & II & n.s. \\
\hline Brasil & 1981 & II & n.s. & 1982 & II & n.s. & 1995 & II & $1 \%$ \\
\hline Chile & 1992 & II & n.s. & 1982 & II & n.s. & 2000 & II & $1 \%$ \\
\hline Colombia & 1975 & II & n.s. & 1992 & II & $5 \%$ & 1990 & I & $5 \%$ \\
\hline Costa Rica & 1974 & II & n.s. & 1981 & II & n.s. & 1990 & II & $1 \%$ \\
\hline Ecuador & 1977 & II & n.s. & 1983 & II & n.s. & 1998 & II & n.s. \\
\hline El Salvador & 1979 & II & $1 \%$ & 1986 & II & n.s. & 2000 & II & n.s. \\
\hline Guatemala & 1981 & I & n.s. & 1981 & II & n.s. & & s.d.s & \\
\hline Honduras & 1980 & II & n.s. & 1997 & II & n.s. & & s.d.s & \\
\hline Jamaica & 1974 & II & $1 \%$ & 1984 & II & n.s. & & s.d.s & \\
\hline México & 1982 & I & $5 \%$ & 1982 & II & n.s. & 1980 & II & $1 \%$ \\
\hline Nicaragua & 1978 & II & $1 \%$ & 1992 & II & n.s. & 1980 & II & $5 \%$ \\
\hline Panamá & 1991 & II & n.s. & 1990 & II & n.s. & 1988 & II & $1 \%$ \\
\hline Paraguay & 1982 & II & $1 \%$ & 1990 & II & n.s. & 1984 & II & n.s. \\
\hline Perú & 1988 & II & n.s. & 1990 & II & n.s. & 1982 & II & n.s. \\
\hline Rep. Dominicana & 1977 & II & n.s. & 1982 & II & $5 \%$ & 1984 & II & n.s. \\
\hline Trinidad y Tobago & 1982 & II & n.s. & 1997 & II & n.s. & & s.d.s & \\
\hline Uruguay & 1982 & II & $1 \%$ & 1992 & II & n.s. & 1979 & II & $1 \%$ \\
\hline Venezuela & 1990 & II & n.s. & 1980 & II & n.s. & 1987 & II & $1 \%$ \\
\hline
\end{tabular}

Fuente: Estimación propia.

s.d.s. Sin datos suficientes.

n.s. No significativo.

El Gráfico 1 muestra la distribución de frecuencia de los potenciales cambios estructurales en el nivel de la PTF y el Gráfico 2 muestra la misma información para el indicador de apertura o intensidad comercial, (X+M)/PIB.

En cuanto a los niveles de PTF se observa una mayor concentración de potenciales quiebres en el período 1974-1982, siendo este último año el de mayor cantidad de casos. Mientras que un segundo conjunto se ubica en el período 1988-1992. Sin 
embargo, los casos significativos se reducen a 1974, 1978 y 1979, cada uno con un caso, y 1982 con tres. La intensidad comercial muestra un conjunto numeroso de casos potenciales en el período 1980-1986, seguido por el período 1990-1992 y 1997. Sin embargo, solo se encuentran dos casos significativos, uno en 1982 y otro en 1992.

\section{GRAFICO 1}

DISTRIBUCION DE POSIBLES QUIEBRES ESTRUCTURALES EN LA PTF

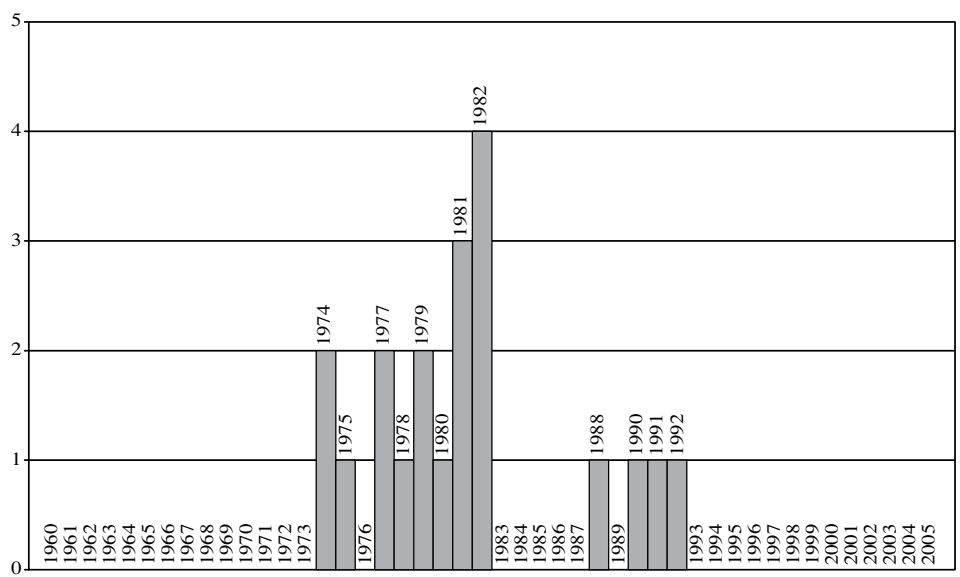

Fuente: Estimación propia.

\section{GRAFICO 2}

DISTRIBUCION DE POSIBLES QUIEBRES ESTRUCTURALES EN LA INTENSIDAD COMERCIAL

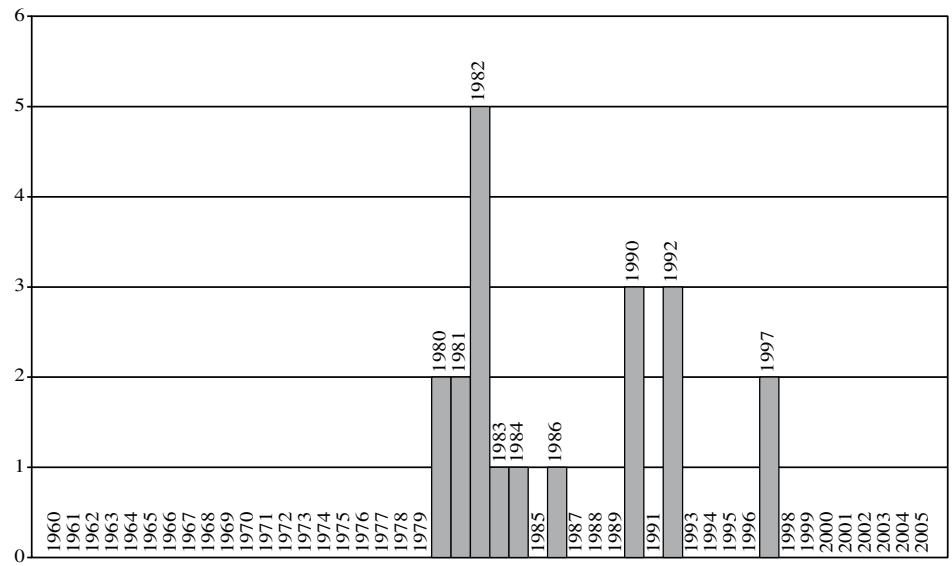

Fuente: Estimación propia. 
Si se realizara un gráfico similar a los anteriores que representara la distribución de los potenciales puntos de quiebre en el indicador de política comercial, 1-r, y en el indicador de desviación, u, el primer gráfico presentaría una distribución relativamente homogénea de los casos potenciales a lo largo del período 1979-2000. De los cuales, son significativos dos casos en 1979-1978, cuatro casos en 1987-1992, dos casos en 1990, y uno en 1995 y 2000, respectivamente. El indicador de desviación, u, concentra la mayor cantidad de casos potenciales en dos períodos: 1980-1984 con doce casos y 1989-1993 con seis casos. Finalmente, el indicador de intensidad comercial alternativo muestra siete casos potenciales en 1979-1983 y cuatro casos en 1993-1995. Los casos significativos son cuatro y tres, respectivamente, sin un patrón de concentración.

Con respecto a la contemporaneidad de los quiebres significativos analizando cada país individualmente, se observa en la Tabla 1 que solo en Colombia existe proximidad entre el indicador de política comercial y el indicador de resultado, aunque ello no se ve reflejado en la PTF, ni siquiera como caso potencial. Mientras que se observan solo tres casos, México, Nicaragua y Uruguay, de contemporaneidad entre los quiebres de las series del indicador de intensidad comercial y de la PTF. Sin embargo, el caso de Nicaragua presenta el sentido temporal contrario al esperado y en el caso de Uruguay la contemporaneidad se redefine permitiendo tres años entre un quiebre y el otro. Si se tienen en cuenta los casos potenciales de quiebres en los niveles de PTF, sólo se agregan a la lista anterior los casos de Panamá y Venezuela (este último en las condiciones definidas para Uruguay).

El test de BP (Bai y Perron) arroja como resultado once casos significativos al $5 \%$ de quiebre estructural en la PTF. En cinco casos el test es coincidente con ZA, a los que suman cinco casos datados entre 1981 y 1983, resta a Uruguay y suma a Venezuela con fecha en 1973. La Tabla 2 muestra estos resultados junto a los obtenidos para los indicadores de apertura (salvo 1-r que no se ha realizado debido a que los datos no son suficientes en la mayor parte de las economías de la muestra). Entre paréntesis aparecen, reiterados en algunos casos, los resultados significativos al menos al 5\% según el test de ZA.

En cuanto a la contemporaneidad entre los quiebres obtenidos a partir de BP en la PTF y en los indicadores de apertura mediante el mismo método, los resultados son aún menos optimistas que los presentados para ZA. Tan solo en el caso de Venezuela se encuentra exacta contemporaneidad en el año 1973, mientras que en Trinidad y Tobago el sentido de la contemporaneidad es el contrario al esperado.

Tres resultados interesantes para mencionar son: Primero, a pesar de permitir la multiplicidad de quiebres estructurales en las series de PTF, el test de BP no presenta ningún caso con más de un cambio estructural significativo en la productividad multifactorial.

Segundo, la existencia de casos con múltiples quiebres en las series de apertura -Argentina, Colombia, entre otros- no garantiza la existencia de contemporaneidad entre los quiebres de los diferentes indicadores. Ello significa que estos no necesariamente están capturando el mismo fenómeno y, por consiguiente, la información que suministran individualmente debe considerarse parcial.

Tercero, solo en los casos (Argentina, 1975-1976), (Argentina, 1993), (Colombia, 1989-1992), (República Dominicana, 1982-1983), (Ecuador, 1971-1972), (El Salvador, 
TABLA 2

QUIEBRES ESTRUCTURALES EN LAS SERIES DE PTF Y LOS INDICADORES DE APERTURA SEGUN EL TEST DE BP

\begin{tabular}{|c|c|c|c|c|c|}
\hline País & PTF & $\begin{array}{c}(\mathrm{X}+\mathrm{M}) / \\
\mathrm{PIB}\end{array}$ & $1-\mathrm{r}$ & $\mathrm{u}$ & $\begin{array}{c}(\mathrm{X}+\mathrm{M}) / \\
\text { PIB* }^{*}\end{array}$ \\
\hline Argentina & & & & $\begin{array}{l}1968 \\
1976 \\
1993\end{array}$ & $\begin{array}{c}1975 \\
1993 \\
1999(1998)\end{array}$ \\
\hline Bolivia & 1982 & & & & \\
\hline Brasil & & & (1995) & & $\begin{array}{l}1971 \\
1999\end{array}$ \\
\hline Chile & & & (2000) & & \\
\hline Colombia & & 1992 & (1990) & $\begin{array}{c}1969 \\
1990(1991)\end{array}$ & 1989 \\
\hline Costa Rica & & & (1990) & & (1983) \\
\hline Ecuador & & & & $\begin{array}{l}1965 \\
1971 \\
1993\end{array}$ & 1972 \\
\hline El Salvador & 1979 (1979) & 1996 & & 1994 & \\
\hline Guatemala & 1981 & & & & 1999 \\
\hline Honduras & 1983 & & & & \\
\hline Jamaica & 1974 (1974) & $\begin{array}{l}1965 \\
1984\end{array}$ & & & 1978 \\
\hline México & $1982(1982)$ & 1994 & (1980) & & (1995) \\
\hline Nicaragua & 1978 (1978) & 1994 & (1980) & 1995 & \\
\hline Panamá & & 1998 & (1988) & 1998 & \\
\hline Paraguay & 1982 (1982) & 1989 & & $\begin{array}{l}1976 \\
1989\end{array}$ & \\
\hline Perú & 1982 & & & & \\
\hline Rep. Dominicana & & $\begin{array}{l}1980 \\
1993\end{array}$ & & (1982) & 1983 \\
\hline Trinidad y Tobago & 1982 & $\begin{array}{l}1968 \\
1983 \\
1996\end{array}$ & & 1994 (1997) & $\begin{array}{l}1965 \\
1994\end{array}$ \\
\hline Uruguay & (1982) & & (1979) & (1992) & \\
\hline Venezuela & 1973 & & (1987) & & $\begin{array}{l}1965 \\
1973\end{array}$ \\
\hline
\end{tabular}

Fuente: Estimación propia.

1994-1996), (México, 1994-1995), (Nicaragua, 1994-1995), (Panamá, 1998), (Paraguay, 1989), (Trinidad y Tobago, 1994-1997), las series de apertura arrojan resultados robustos de cambio estructural en el comercio internacional entre estos países y el resto del mundo. Sin embargo, aun en estos casos no existe contemporaneidad entre estos quiebres estructurales y los obtenidos para la PTF. 
Finalmente, se comparan los quiebres estructurales en los niveles de PTF con los cambios de política comercial que son registrados en la literatura especializada. El objeto es robustecer los resultados de los tests sobre las medidas de apertura. La Tabla 3 presenta una periodización de la política comercial para cada país de la muestra.

\section{TABLA 3}

\section{SUMARIO DE QUIEBRES POTENCIALES PRESENTADOS EN LA LITERATURA ESPECIALIZADA}

\begin{tabular}{|c|c|c|c|}
\hline País & $\begin{array}{l}\text { Año o } \\
\text { período }\end{array}$ & $\begin{array}{l}\text { Carácter de la política } \\
\text { comercial }\end{array}$ & Fuente de la periodización \\
\hline Argentina & $\begin{array}{l}1930-1976 \\
1976-1981 \\
1981-1988 \\
1988-1991 \\
1991\end{array}$ & $\begin{array}{l}\text { Cierre } \\
\text { Apertura } \\
\text { Cierre } \\
\text { Apertura } \\
\text { Consolidación de la Apertura }\end{array}$ & Hopenhayn y Neumeyer (2005) \\
\hline Bolivia & $\begin{array}{l}1950-1985 \\
1986\end{array}$ & $\begin{array}{l}\text { Cierre } \\
\text { Apertura }\end{array}$ & $\begin{array}{l}\text { Zambrana Calvimonte (2002), } \\
\text { Agosin y Ffrench-Davis (1994) }\end{array}$ \\
\hline Brasil & $\begin{array}{l}1930-1980 \\
1981-1993 \\
1994\end{array}$ & $\begin{array}{l}\text { Cierre } \\
\text { Apertura } \\
\text { Consolidación de la Apertura }\end{array}$ & Castelar Pinheiro et al. (2005) \\
\hline Chile & $\begin{array}{l}1930-1959 \\
1959-1961 \\
1961-1973 \\
1970-1973 \\
1974-1979 \\
1979-1984 \\
1985-1991\end{array}$ & $\begin{array}{l}\text { Cierre } \\
\text { Apertura } \\
\text { Cierre } \\
\text { Profundización del Cierre } \\
\text { Apertura } \\
\text { Apertura moderada } \\
\text { Consolidación de la Apertura }\end{array}$ & $\begin{array}{l}\text { Chumancero y Fuentes (2005), } \\
\text { Agosin y Ffrench-Davis } \\
\text { (1994). Santos Paulinos y } \\
\text { Thirlwall (2006) señalan } \\
\text { a 1976 como el año de la } \\
\text { liberalización del comercio. }\end{array}$ \\
\hline Colombia & $\begin{array}{l}1930-1967 \\
1967-1981 \\
1982-1985 \\
1985-1989 \\
1990\end{array}$ & $\begin{array}{l}\text { Cierre } \\
\text { Apertura } \\
\text { Cierre } \\
\text { Apertura } \\
\text { Consolidación de la Apertura }\end{array}$ & $\begin{array}{l}\text { Ocampo y Villar (1992), } \\
\text { Santos Paulinos y Thirlwall } \\
\text { (2004) señalan a 1991 como } \\
\text { año de la liberalización del } \\
\text { comercio. }\end{array}$ \\
\hline Costa Rica & $\begin{array}{l}1962-1986 \\
1986-1989 \\
1989\end{array}$ & $\begin{array}{l}\text { Cierre } \\
\text { Apertura } \\
\text { Consolidación de la Apertura }\end{array}$ & $\begin{array}{l}\text { Cordero (2000), Santos } \\
\text { Paulinos y Thirlwall (2004) } \\
\text { señalan a } 1990 \text { como año de la } \\
\text { liberalización del comercio. }\end{array}$ \\
\hline Ecuador & $\begin{array}{l}1960-1989 \\
1989-1995\end{array}$ & $\begin{array}{l}\text { Cierre } \\
\text { Apertura }\end{array}$ & $\begin{array}{l}\text { Wong (2007), Santos Paulinos } \\
\text { y Thirlwall (2004) señalan } \\
\text { a } 1991 \text { como año de la } \\
\text { liberalización del comercio. }\end{array}$ \\
\hline El Salvador & $\begin{array}{l}1960-1989 \\
1989-1995 \\
1996\end{array}$ & $\begin{array}{l}\text { Cierre } \\
\text { Apertura } \\
\text { Consolidación de la Apertura }\end{array}$ & $\begin{array}{l}\text { Jul et al. (1998), Dijstra } \\
\text { (1995), Paus (1995) }\end{array}$ \\
\hline Guatemala & $\begin{array}{l}1951-1982 \\
1986-1995 \\
1995\end{array}$ & $\begin{array}{l}\text { Cierre } \\
\text { Apertura } \\
\text { Consolidación de la Apertura }\end{array}$ & Cuevas y Bolaños (2007) \\
\hline
\end{tabular}


TABLA 3 (Continuación)

\begin{tabular}{|c|c|c|c|}
\hline País & $\begin{array}{l}\text { Año o } \\
\text { período }\end{array}$ & $\begin{array}{l}\text { Carácter de la política } \\
\text { comercial }\end{array}$ & Fuente de la periodización \\
\hline Honduras & $\begin{array}{l}1960-1989 \\
1990-1994 \\
1994\end{array}$ & $\begin{array}{l}\text { Cierre } \\
\text { Apertura } \\
\text { Consolidación de la Apertura }\end{array}$ & De Hoyos et al. (2008) \\
\hline Jamaica & $\begin{array}{r}1962-1977 \\
1977-1984 \\
1985-1991 \\
1991-1996\end{array}$ & $\begin{array}{l}\text { Cierre } \\
\text { Apertura moderada } \\
\text { Apertura } \\
\text { Consolidación de la Apertura }\end{array}$ & $\begin{array}{l}\text { Charlton (1992), Peltzman } \\
\text { (2004) }\end{array}$ \\
\hline México & $\begin{array}{l}1940-1982 \\
1982-1993 \\
1993\end{array}$ & $\begin{array}{l}\text { Cierre } \\
\text { Apertura } \\
\text { Consolidación de la Apertura }\end{array}$ & $\begin{array}{l}\text { Romero (2003), Agosin y } \\
\text { Ffrench-Davis (1994) señalan } \\
\text { el inicio de la consolidación de } \\
\text { la apertura en 1985, y Santos } \\
\text { Paulinos y Thirlwall (2004) } \\
\text { señalan a } 1986 .\end{array}$ \\
\hline Nicaragua & $\begin{array}{l}1960-1985 \\
1985-1988 \\
1988-1990 \\
1991\end{array}$ & $\begin{array}{l}\text { Cierre } \\
\text { Apertura } \\
\text { Apertura moderada } \\
\text { Apertura }\end{array}$ & Spoor $(1995)$ \\
\hline Panamá & $\begin{array}{l}1960-1991 \\
1991-1994 \\
1994\end{array}$ & $\begin{array}{l}\text { Dual } \\
\text { Apertura } \\
\text { Consolidación de la Apertura }\end{array}$ & De Jong y Vos (2000) \\
\hline Paraguay & $\begin{array}{l}1960-1975 \\
1975-1981 \\
1981\end{array}$ & $\begin{array}{l}\text { Cierre } \\
\text { Apertura } \\
\text { Consolidación de la Apertura }\end{array}$ & $\begin{array}{l}\text { Masi (2006), Santos Paulinos } \\
\text { y Thirlwall (2004) señalan } \\
\text { a } 1986 \text { como año de la } \\
\text { liberalización del comercio. }\end{array}$ \\
\hline Perú & $\begin{array}{l}1950-1965 \\
1966-1990 \\
1990\end{array}$ & $\begin{array}{l}\text { Apertura } \\
\text { Cierre } \\
\text { Consolidación de la Apertura }\end{array}$ & Carranza et al. (2005) \\
\hline Rep. Dominicana & $\begin{array}{l}1960-1989 \\
1990-1992\end{array}$ & $\begin{array}{l}\text { Cierre } \\
\text { Apertura }\end{array}$ & $\begin{array}{l}\text { Santos Paulinos (2006), Santos } \\
\text { Paulinos y Thirlwall (2004) }\end{array}$ \\
\hline Trinidad y Tobago & $\begin{array}{l}1960-1988 \\
1989-1994 \\
1994\end{array}$ & $\begin{array}{l}\text { Cierre } \\
\text { Apertura } \\
\text { Consolidación de la Apertura }\end{array}$ & $\begin{array}{l}\text { Dobson y Ramlogan (2001), } \\
\text { Kazarian y Ames (2000) }\end{array}$ \\
\hline Uruguay & $\begin{array}{l}1930-1959 \\
1959-1963 \\
1963-1973 \\
1973\end{array}$ & $\begin{array}{l}\text { Cierre } \\
\text { Apertura } \\
\text { Cierre } \\
\text { Apertura }\end{array}$ & $\begin{array}{l}\text { De Brun (2005), Santos } \\
\text { Paulinos y Thirlwall (2004) } \\
\text { señalan a } 1985 \text { como año de la } \\
\text { liberalización del comercio. }\end{array}$ \\
\hline Venezuela & $\begin{array}{l}1960-1989 \\
1989-1992 \\
1993-1996 \\
1996\end{array}$ & $\begin{array}{l}\text { Dual } \\
\text { Apertura } \\
\text { Apertura moderada } \\
\text { Apertura }\end{array}$ & $\begin{array}{l}\text { Corrales y Cisneros (1999), } \\
\text { Santos Paulinos y Thirlwall } \\
\text { (2004) señalan a } 1991 \text { como } \\
\text { año de la liberalización del } \\
\text { comercio. }\end{array}$ \\
\hline
\end{tabular}

Fuente: Elaboración propia. 
De la información suministrada en el cuadro anterior y de los quiebres significativos encontrados en los indicadores de apertura en todas sus versiones, se desprende que sólo en Argentina (BP), Brasil (ZA), Colombia (ZA y BP), Costa Rica (ZA), El Salvador (BP), Jamaica (BP), México (BP), Paraguay (BP), y Trinidad y Tobago (BP) existe contemporaneidad entre el resultado de los tests realizados sobre las series de apertura y un cambio de política resaltado en la literatura. Mientras que sólo en tres casos existe contemporaneidad entre un cambio resaltado en la literatura y un quiebre significativo en los niveles de la PTF (Guatemala, México y Paraguay).

Sintetizando, teniendo en cuenta únicamente los resultados de los tests ZA y BP existiría contemporaneidad entre cambios abruptos en la política comercial y cambios significativos en los niveles de PTF, en los casos (México, 1980-1982), (Uruguay, 1979-1982) y (Venezuela, 1973). A los que podría sumarse (Paraguay, 1981-1982) si se tiene en cuenta la literatura consultada, y (Trinidad y Tobago, 1983-1982) y (Guatemala, 1982-1981), ambos con el sentido inverso al esperado.

La Tabla 4 muestra los quiebres significativos al menos al 5\% en las series de tasas de crecimiento de la PTF, tomada ésta como la diferencia en logaritmos de las series de niveles, utilizando los test de ZA y BP.

La primera observación que puede hacerse es la coexistencia en la mayoría de los casos de quiebres significativos en dos o en tres de los modelos propuestos por el test ZA. Por el criterio mencionado en las Sección 2 es preferible el modelo III ante esta situación. A pesar del resultado anterior, solo en el caso (México, 1987) existe coincidencia entre los test.

La segunda es que en seis casos existe contemporaneidad entre quiebres en niveles y quiebres en tasas de crecimiento de la PTF (Colombia, Ecuador, Guatemala, Honduras, Panamá, Trinidad y Tobago), pero ninguno corresponde a los casos de contemporaneidad entre quiebres de apertura y niveles. Esto significa que la contemporaneidad de quiebres en las series de apertura y en niveles de PTF no asegura la existencia de quiebres contemporáneos en la serie de tasas de crecimiento de la PTF.

\section{TABLA 4}

QUIEBRES ESTRUCTURALES EN LAS SERIES DE TASAS DE CRECIMIENTO DE LA PTF SEGUN LOS TESTS ZA Y BP

\begin{tabular}{|l|c|c|c|c|}
\hline \multicolumn{2}{|c|}{ País } & \multicolumn{3}{|c|}{ gPTF } \\
\cline { 2 - 5 } \multicolumn{1}{|c|}{ ZA } & BP \\
\hline Argentina & Modelo I & Modelo II & Modelo III & \\
Bolivia & 1991 & 1982 & 1991 & \\
Brasil & 1968 & 1969 & 1968 & 1966 \\
Chile & & & & \\
Colombia & 1984 & 1993 & 1976 & 1971 \\
Costa Rica & 1974 & 1977 & 1975 & 1998 \\
\hline
\end{tabular}


TABLA 4 (Continuación)

\begin{tabular}{|c|c|c|c|c|}
\hline \multicolumn{5}{|c|}{ gPTF } \\
\hline \multirow{2}{*}{ País } & \multicolumn{3}{|c|}{$\mathrm{ZA}$} & \multirow[t]{2}{*}{ BP } \\
\hline & Modelo I & Modelo II & Modelo III & \\
\hline Ecuador & 1976 & 1983 & 1977 & $\begin{array}{l}1973 \\
1982\end{array}$ \\
\hline El Salvador & & & & 1973 \\
\hline Guatemala & & 1982 & 1984 & 1969 \\
\hline Honduras & 1969 & 1983 & 1980 & $\begin{array}{l}1965 \\
1998\end{array}$ \\
\hline \multicolumn{5}{|l|}{ Jamaica } \\
\hline México & 1969 & 1983 & 1987 & $\begin{array}{l}1967 \\
1987\end{array}$ \\
\hline \multicolumn{5}{|l|}{ Nicaragua } \\
\hline Panamá & 1989 & 1975 & 1989 & 1966 \\
\hline Paraguay & 1980 & & & \\
\hline Perú & 1991 & & & \\
\hline Dominicana, Rep. & 1974 & 1979 & 1987 & \\
\hline Trinidad y Tobago & 1971 & 1985 & 1983 & \\
\hline Uruguay & 1986 & 1983 & 1986 & \\
\hline Venezuela & 1998 & 1968 & 1998 & \\
\hline
\end{tabular}

Fuente: Estimación propia.

$g T F P_{t}=\ln T F P_{t}-\ln T F P_{t-1}$

La tercera observación es que la distribución de quiebres significativos es similar entre los períodos 1973-1984 y 1985-1995 (ocho y seis casos, respectivamente, de acuerdo a ZA, mientras que tres y tres siguiendo a BP), y no existe un año que concentre más de dos casos. Finalmente, el test de BP arroja seis casos en los que los quiebres en las series de tasas de crecimiento de la PTF se encuentran entre 1966 y 1971.

La cuarta observación es que son nueve los casos de contemporaneidad entre los quiebres en las series de apertura remarcados en la literatura y estimados mediante ZA, y quiebres en la serie de tasas de crecimiento de la PTF: (Argentina, 1991), (Chile, 1976), (Costa Rica, 1981-1983), (República Dominicana, 1984-1987), (Guatemala, 1981-1984), (México, 1986-1987), (Panamá, 1988-1989), (Perú, 1990-1991) y (Uruguay, 1985-1986). En cambio, mediante el test de BP solo en dos casos existe contemporaneidad (Colombia, 1969-1971) y (Ecuador, 1972-1973). La multiplicidad de coincidencias muestra un marcado contraste con el obtenido para los niveles de PTF.

\section{COMENTARIOS FINALES}

Se ha estudiado la existencia de quiebres estructurales en la PTF, tanto en niveles como en tasas de crecimiento, y en varios indicadores de apertura para una muestra 
de veinte economías latinoamericanas y del Caribe y el período comprendido entre 1960 y 2005. Para ello se ha utilizado el test de Zivot y Andrews (1992) y el test de Bai y Perron (1998) sobre una serie de PTF computada a partir de un ejercicio de contabilidad del desarrollo (Hsieh y Klenow, 2010) y sobre cuatro medidas alternativas de apertura con diferentes caracteres: medidas de política, de resultado y de desvío (Wacziarg, 2001).

Se puede afirmar que entre 1980 y 2000 prácticamente todas las economías experimentaron un cambio profundo de política comercial hacia una consolidación de la apertura. Los quiebres estructurales que se desprenden a partir de la aplicación del test ZA marcan claramente esta situación en doce economías de las veinte consideradas, mientras que trece son las economías siguiendo a los resultados del test de BP.

Sin embargo, no se observa, salvo en contados casos, que exista contemporaneidad entre los shocks por la apertura y los shocks en niveles de la PTF. Esto estaría indicando que los shocks observados en el nivel de la PTF, en general, no serían consecuencia de los cambios abruptos en las políticas comerciales (tanto cierre como apertura), en cambio tendrían otros orígenes.

La acumulación de casos, tanto potenciales como significativos, de quiebres en los niveles de PTF coincide con las Crisis del Petróleo de 1973/1974 y 1979/1980, y la Crisis Mexicana de 1982. Por otra parte, los casos de quiebre de niveles de la PTF durante el proceso de consolidación de la apertura en la región (1985-1995) no son significativos en el caso de ZA y no existen según el test de BP.

Los casos de contemporaneidad en los quiebres de las series de apertura y tasas de crecimiento son más numerosos siguiendo ZA, sin embargo su existencia no es contemporánea con un cambio estructural en el nivel de la PTF, al menos para la muestra y rango temporal considerado. El resultado del test de BP es aún menos optimista. Solo en dos casos existiría contemporaneidad entre apertura y tasa de crecimiento en la PTF, sin embargo los años de ocurrencia son anteriores al período de apertura, y tampoco existiría un correlato con los niveles de la PTF.

Un posible ejercicio que podría controlar los pocos quiebres hallados sería testear en conjunto con una estructura que explique el comportamiento de las series utilizadas, es decir, realizar un test de quiebre parcial de BP que complemente el test de quiebre puro que se ha realizado. En el presente ejercicio se ha evitado suponer una forma funcional para concentrar el análisis en la contemporaneidad de las series tomadas uniecuacionalmente.

Finalmente, los resultados para la muestra considerada y bajo la especificación utilizada parecen indicar que, luego de un proceso abrupto de apertura (o cierre), los cambios en la PTF, de existir, son graduales. Si bien es preciso realizar otros ejercicios para testear causalidad (y el sentido de la misma), las recomendaciones de política que se desprenden de estos resultados apuntan hacia cambios graduales de la apertura en lugar de aperturas unilaterales y de una vez, debido a que los beneficios provenientes de las ganancias de productividad no compensarían inmediatamente los costos asociados con la reestructuración productiva que acompaña a un cambio abrupto de política comercial. 


\section{REFERENCIAS}

AGOSIN, M. y R. FFRENCH-DAVIS (1997). "Financial Liberalization and Development. A view form emerging economies", Estudios de Economia 24 (2), pp. 207-217.

ALCALA, F. y A. CICCONE (2004). "Trade and productivity", Quarterly Journal of Economics 119 (2), pp. 613-646.

ANDERSON, J. y P. NEARY (1992). "A new approach to evaluating trade policy", World Bank Policy Research Working Papers in International Trade, WPS1022.

BAI, J. y P. PERRON (1998). "Estimating and Testing Linear Models with Multiple Structural Changes", Econometrica 66, pp. 47-78.

BAI, J. y P. PERRON (2003). "Computation and Analysis of MultipleStructural Change Models", Journal of Applied Econometrics 18, pp. 1-22.

BALDWIN, R. (2003). “Openness and growth. What's the empirical relationship?” NBER Working paper, 9578.

BENJAMIN, N. y M. FERRANTINO (2001). "Trade policy and productivity growth in OECD manufacturing”, International Economic Journal 15 (4), pp. 95-115.

CALVANTI FERREIRA, P. y A. TREJOS (2006). "Measuring the TFP costs of barriers to trade", EPGE Working paper, april.

CARRANZA, E., J. FERNANDEZ-BACA y E. MORON (2005). "Markets, government, and the sources of Growth in Peru", en Fernández-Arias, E., R. Manuelli y J. Blyde, Sources of Growth in Latin America. What is missing?, Inter-American Development Bank, chapter 7.

CASTELAR PINHEIRO, A., I. GILL, L. SERVEN y M. THOMAS (2005). "Brazilian Economic Growth, 1900-2000. Lessons and policy implications", en Sources of Growth in Latin America. What is missing?, en Fernández-Arias, E., R. Manuelli y J. Blyde, Inter-American Development Bank, chapter 4.

CHARLTON, C. (1992). "Investment patterns and economic growth in Jamaica. 1981-1988”, ISS Working paper 118 .

CHUMANCERO, R. y R. FUENTES (2005). "On the determinants of Chilean Economic Growth" en Fernández-Arias, E., R. Manuelli y J. Blyde, Sources of Growth in Latin America. What is missing?, Inter-American Development Bank, chapter 5.

CORDERO, J. (2000). "El crecimiento económico y la inversión. El caso de Costa Rica" Serie Reformas Económicas 52.

CORRALES, J. e I. CISNEROS (1999). "Corporatism, Trade Liberalization and Sectoral Responses. The Case of Venezuela, 1989-99”, World Development 27 (12), pp. 2099-2122.

CUEVAS, M. y L. BOLAÑOS (2007). "Opportunities and risks in the liberalisation of trade in services. The case of Guatemala”, MPRA Paper 9941.

DAS, D. (2002). "Trade liberalization and industrial productivity. An assessment of developing country experiences", Indian Council for Research on International Economic Relations working paper 77.

DE BRUN, J. (2005). "Growth in Uruguay. Factor acumulation or productivity gains", en Fernández-Arias, E., R. Manuelli y J. Blyde, Sources of Growth in Latin America. What is missing?, Inter-American Development Bank, chapter 8.

DE HOYOS, R., M. BUSSOLO y O. NUÑEZ (2008). “Can Maquila booms reduce poverty? Evidence from Honduras", World Bank Policy Research Working Paper 4789.

DE JONG, N. y R. VOS (2000). "Economic reforms and rising inequality in Panama in the 1990s", ISS Working paper 318.

DIJSTRA, G. (1995). "The benefits of economic integration. The case of Central America", ISS Working paper 207.

DICKEY, D. y W. FÜLLER (1984). “Testing for unit roots in seasonal time series”. Journal of the American Statistical Associations 79, pp. 355-367.

DOBSON, S. y C. RAMLOGAN (2001). "Money Demand and Economic Liberalization in a Small Open Economy-Trinidad and Tobago", Open Economies Review 12, pp. 325-339.

EDWARDS, S. (1998). “Openness, productivity and growth. what do we really know?", Economic Journal 108, pp. 383-398.

GONZALEZ, G. (2002). "Apertura, Orientación Comercial y Productividad Total de los Factores. La incidencia del nivel de desarrollo", Anales de la Asociación Argentina de Economía Política. 
GONZALEZ, G., M. DABUS y P. MONTERUBBIANESI (2009). "Convergencia en economías semiindustrializadas. Nueva evidencia de América Latina y Caribe", Anales de la Asociación Argentina de Economía Política.

GONZALEZ G. y S. CONSTANTIN (2009). "Efectos de la apertura sobre el crecimiento cuando se tiene en cuenta el contexto", Revista Ciencias Económicas 27 (2), pp. 11-20.

HARBERGER, A. (1998). "A vision of growth process", American Economic Review 88, pp. 1-32.

HAOUAS, I. y M. YAGOUBI (2005). "Openness and human capital as sources of productivity growth. An empirical investigation from the MENA countries" IZA discussion paper 1461.

HERZER, D. (2005). "Does trade increase total factor productivity. Cointegration evidence for Chile", IAI Working paper 115.

HOPENHAYN, H. y A. NEUMEYER (2004). "Latin America in the XXth Century. Stagnation, then Collapse" en Fernández-Arias, E., R. Manuelli y J. Blyde, Sources of Growth in Latin America. What is missing?, Inter-American Development Bank, Part III.

HOPENHAYN, H. y A. NEUMEYER (2005). "Explaining Argentina's Great Depression of 1975-90" en Fernández-Arias, E., R. Manuelli y J. Blyde, Sources of Growth in Latin America. What is missing?, Inter-American Development Bank, Chapter 3.

HSIEH, CH. y P. KLENOW (2010). “Development Accounting.” American Economic Journal Macroeconomics 2 (1), pp. 207-223.

JONSSON, G. y A. SUBRAMANIAN (2001). "Dynamic gains from trade. Evidence from South Africa", IMF Working paper 45.

JUL, A., F. FRANTISCHEK, J. MIKKELSEN y R. MORALES (1998). "El Salvador. Recent Economic Developments", IMF Staff Country Report 98/32.

KAZARIAN, N. y G. AMES (2000). "Trade liberalization in Trinidad and Tobago. Reducing the Common External Tariff", Dept. of Agricultural and Applied Economics, University of Georgia, Working paper.

KLENOW, P. y A. RODRIGUEZ-CLARE (1997). "The Neoclassical Revival in Growth Economics. Has it Gone too far?, en Bernanke B. y J. Rotermberg, NBER Macroeconomics Annual, pp. 73-103.

KNELLER, R., C. MORGAN y S. KANCHANAHATAKIJ (2008). "Trade Liberalisation and Economic Growth", The World Economy 31 (6), pp. 701-719.

KRISHNA, K. (1991). "Openness. A conceptual approach", Harvard University working paper.

LALL, S. (1992). "Technological Capabilities and Industrialization", World Development 20 (2), pp. 165-186.

MANKIW, N., D. ROMER y D. WEIL (1992). "A contribution to the empirics of economic growth", Quarterly Journal of Economics 107 (2), pp. 407-437.

MASI, F. (2006). "Paraguay. Los vaivenes de la política comercial externa en una economía abierta", CADEP working paper.

McGRATTAN, E. y J. SCHMITZ (1999). "Explaining cross-country income differences", en Taylor J. y Woodford M. Handbook of Macroeconomics, Elsevier, Volume 1.

MILLER, S. y M. UPADHYAY (2000). "The effects of openness, trade orientation, and human capital on total factor productivity", Journal of Development Economics 63, pp. 399-423.

MILLER, S. y M. UPADHYAY (2002). "Total factor productivity, human capital, and outward orientation. Differences y stage of development and geographic regions", University of Nevada and Eastern Illinois University working paper.

NEHRU, V. y A. DHARESHWAR (1993). "A New Database on Physical Capital Stock. Sources, Methodology and Results", Revista de Análisis Económico 8 (1), pp. 37-59.

OCAMPO J. y L. VILLAR (1992). "Trayectorias y vicisitudes de la apertura económica colombiana", Pensamiento Iberoamericano 21, pp. 165-86.

PAUS, E. (1995). "Exports, economic growth and the consolidation of peace in El Salvador", World Development 23 (12), pp. 2173-2193.

PELTZMAN, J. (2004). "Trade liberalization and fiscal reform. Evidence from two case studies -Morocco and Jamaica- and a general cross-country econometric analysis", United States Agency for International Development, October.

PERRON, P. (1989). "The great crash, the oil price shock and the unit root hypothesis", Econometrica 57, pp. 1361-1401.

PRITCHETT, L. (1996). "Measuring outward orientation in LDCs. can it bi done?" Journal of Development Economics 49, pp. 307-335. 
RODRIGUEZ, F. y D. RODRIK (2000). "Trade policy and economic growth. A skeptic's guide to the cross-national evidence", en Bernanke, B. y K. Rogoff, NBER Macroeconomics Annual 2000, MIT Press.

RODRIK, D. (2003). “Introduction”, en Rodrik, D. In Search of Prosperity. Analytic Narratives on Economic Growth, Princeton University Press.

SANTOS PAULINOS, A. (2006). "Trade liberalization and trade performance in the Dominican Republic", Journal of International Development 18, pp. 925-944.

SANTOS PAULINOS, A. y A. THIRLWALL (2004). "The impact of trade liberalization on exports, imports and the balance of payments of developing countries", Economic Journal 114, F50-F72.

SEN, A. (2003). "On unit root tests when the alternative is a trend break stationary process", Journal of Business and Economic Statistics 21, pp. 174-184.

SÖDERBOM, M. y F. TEAL (2003). "Openness and human capital as sources of productivity growth. An empirical investigation", CSAE Working paper 06.

SPOOR, M. (1995). "Liberalization of grain markets in Nicaragua. From market substitution to state minimalism", Food Policy 20 (2), pp. 99-110.

WACZIARG, R. (2001). "Measuring the dynamic gains from trade", World Bank Economic Review 15 (3), pp. 393-429.

WONG, S. (2007). "Productivity and trade openness. Micro-level evidence from manufacturing industries in Ecuador 1997-2003", APEA 2007 Conference.

ZAMBRANA CALVIMONTE, H. (2002). "La apertura externa en Bolivia", Serie Análisis Económico, UDAPE.

ZIVOT, E. y D. ANDREWS (1992). "Further Evidence on the Great Crash, the Oil-Price Shock and the Unit-Root Hypothesis", Journal of Business and Economic Statistics 10 (3), pp. 251-270. 


\section{ANEXOS}

\section{Estimación y Fuentes de datos}

\begin{tabular}{|c|c|}
\hline Variable & Especificación \\
\hline $\mathrm{Y}, \mathrm{X}, \mathrm{M}$ & $\begin{array}{l}\text { PIB, exportaciones e importaciones a precios constantes del } 2000 \text { y corrientes, US\$. } \\
\text { Fuente: World Bank, WORLD DATABANK, Noviembre } 2009 .\end{array}$ \\
\hline $\mathrm{P}$ & $\begin{array}{l}\text { Población total. } \\
\text { Fuente: World Bank Development Indicator } 2000 .\end{array}$ \\
\hline $\mathrm{L}$ & $\begin{array}{l}\text { Empleados } \\
\text { Método de cálculo: (1-U/100)* } L F \\
\text { donde } L F \text { es población en edad de trabajar y } U \text { es la tasa de desempleo. } \\
\text { Para los casos en que faltaron períodos extensos se supuso una relación lineal entre la } \\
\text { tasa de crecimiento del producto y la tasa de crecimiento del empleo, y a partir de los } \\
\text { parámetros estimados (mediante OLS y tomando cada caso en particular) se computaron } \\
\text { las tasas de crecimiento del empleo faltantes. Estas fueron aplicadas sobre los niveles } \\
\text { de L para completar la serie. } \\
\text { Fuentes: } \\
\text { 1. Marcel P. Timmer and Gaaitzen J. de Vries (2007). "A Cross-Country Database For } \\
\text { Sectoral Employment And Productivity In Asia And Latin America, 1950-2005", } \\
\text { Groningen Growth and Development Centre Research Memorandum GD-98, } \\
\text { Groningen: University of Groningen, August 2007. } \\
\text { 2. The Conference Board, Total Economy Database, June 2009. } \\
\text { 3. ILO, LABORSTA Labour Statistics Database. } \\
\text { 4. ILO, ICMT 5ta edición. } \\
\text { 5. Tasa de desempleo sobre la fuerza total de trabajo: (i) CEPAL, CEPALSTAT } \\
\text { (ii) ILO, LABORSTA Labour Statistics Database (iii) WBDI } 2007 \text { y (iv) WB } \\
\text { DATABANK. }\end{array}$ \\
\hline K & $\begin{array}{l}\text { Stock de capital físico } \\
\text { Método de cálculo: Construido utilizando la metodología "preferida" de King, R., y } \\
\text { R. Levine (1994): "Capital Fundamentalism, Economic Development, and Economic } \\
\text { Growth", Policy Research Working Paper, } 1285 \text {. The World Bank. } \\
\text { Para estimar el } K / Y \text { inicial en el estado estacionario se utilizaron: la tasa de inversión } \\
\text { promedio para todo el período, las tasas de crecimiento del producto para la economía } \\
\text { y la muestra para todo el período. Los valores de los parámetros son los utilizados por } \\
\text { estos autores: } \lambda=0.25 \text { y } \delta=0.07 \text {. } \\
\text { Estimación propia en base a los siguientes datos: } \\
\text { Fuentes: } \\
\text { 1. Gross fixed capital formation (constant } 2000 \text { US } \$ \text { ), WB DATABANK. } \\
\text { 2. CEPAL, Estadísticas e Indicadores Económicos [BADECON]. } \\
\text { 3. Heston A., R. Summers y B. Aten, Penn World Table Version } 6.2 \text {, Center for } \\
\text { International Comparisons of Production, Income and Prices at the University of } \\
\text { Pennsylvania, September } 2006 \text {. }\end{array}$ \\
\hline
\end{tabular}




\begin{tabular}{|c|c|}
\hline Variable & Especificación \\
\hline $\mathrm{H} / \mathrm{Y}$ & $\begin{array}{l}\text { Intensidad del capital humano } \\
\text { Método de cálculo: } \\
\text { Construido siguiendo a Mankiw, N., D. Romer y D. Weil, (1992). "A contribution to the } \\
\text { empirics of economic growth". Quarterly Journal of Economics, 107, 2, 407-437: } \\
\frac{H}{Y}=\frac{I_{H} / Y}{n+g_{s t}+\delta} \\
\text { donde el numerador es la tasa de inversión en capital humano y fue aproximada } \\
\text { mediante el cociente entre la población enrolada en escuelas de nivel secundario y la } \\
\text { población en edad de trabajar. Los valores de los parámetros son los mismos que en } \\
\text { la estimación de } K \text {. } \\
\text { Fuentes: } \\
\text { 1. Alumnos del secundario, Ferreres, O. Dos Siglos de Economía Argentina (1810- } \\
\text { 2004), Fundación Norte y Sur. } \\
\text { 2. Alumnos del secundario, Della Paolera, G. and A. Taylor (2003). A New Economic } \\
\text { History of Argentina, Cambridge University Press., apéndice. } \\
\text { 3. Alumnos del secundario, CEPAL, CEPALSTAT. } \\
\text { 4. Oxford Latin America Economic History Database. } \\
\text { 5. UNESCO, Institute for Statistics. } \\
\text { 6. Secondary enrolment by level (UNESCO estimates) [code 25540], UNESCO. } \\
\text { 7. Secondary education, pupils WBDI } 2007 \text {. } \\
\text { 8. Secondary enrolment by level BANKS dataset 2005. } \\
\text { 9. Población entre } 15 \text { y } 64 \text { años y Población total, WBDI } 2007 \text {. }\end{array}$ \\
\hline $1-\mathrm{r}$ & $\begin{array}{l}\text { Apertura como medida de política comercial } \\
\text { Método de cálculo: La tasa } r \text { se computa como el cociente entre la recaudación total } \\
\text { tributaria por gravámenes a las transacciones internacionales (exportaciones e impor- } \\
\text { taciones) y el total del comercio internacional (exportaciones más importaciones). } \\
\text { Fuentes: } \\
\text { 1. Global Development Network and World Bank, Macroeconomic dataset. } \\
\text { 2. WB DATABANK. } \\
\text { 3. IMF, GFS dataset. } \\
\text { 4. CEPAL, CEPALSTAT. }\end{array}$ \\
\hline $\mathrm{u}$ & $\begin{array}{l}\text { Apertura como medida de desvío } \\
\text { Método de cálculo: Siguiendo la metodología de estimación presentada en Pritchet } \\
\text { (op. cit.), u es el residuo del siguiente modelo empírico: } \\
\frac{(X+M)}{Y}=a+b P+c \ln S U P+d^{*} \frac{Y}{P}+e^{*} O I L+f^{*} I S L A+\text { residuo } \\
\text { Fuentes: Estimación propia a partir de datos mencionados anteriormente y superficie } \\
\text { (SUP) y dummies (OIL e ISLA) extraídas de CEPII y WBDI } 2007 \text {. }\end{array}$ \\
\hline
\end{tabular}




\section{Test de Dickey-Füller}

\begin{tabular}{|l|l|c|c|}
\hline \multirow{2}{*}{ Argentina } & \multicolumn{3}{|c|}{ PTF } \\
\cline { 2 - 4 } Bolivia & tendencia & intercepto & sin constante \\
Brasil & -2.292 & -1.901 & -0.321 \\
Chile & $-3.215^{* * *}$ & -0.632 & -0.793 \\
Colombia & -1.181 & -1.314 & -0.297 \\
Costa Rica & -1.605 & -1.736 & 0.088 \\
Ecuador & -2.961 & -0.847 & -0.206 \\
El Salvador & -1.633 & -1.002 & -0.205 \\
Guatemala & -1.824 & -0.754 & -0.438 \\
Honduras & -2.432 & -0.116 & -0.815 \\
Jamaica & -2.99 & -0.145 & -0.553 \\
México & -3.016 & -0.307 & -0.858 \\
Nicaragua & -1.329 & -1.048 & -1.196 \\
Panamá & -3.191 & -0.533 & -0.394 \\
Paraguay & -2.642 & -0.353 & -0.789 \\
Perú & -2.738 & -1.126 & -0.187 \\
Rep. Dominicana & -2.514 & 0.085 & -0.75 \\
Trinidad y Tobago & -2.175 & -0.795 & -0.512 \\
Uruguay & -2.202 & -1.536 & -0.32 \\
Venezuela & -2.124 & -0.923 & 0.006 \\
\hline
\end{tabular}

*** significativo al $10 \%$.

\begin{tabular}{|l|l|c|c|l|l|c|}
\hline & \multicolumn{3}{|c|}{$(\mathrm{X}+\mathrm{M}) / \mathrm{PIB}$} & \multicolumn{3}{c|}{$(\mathrm{X}+\mathrm{M}) / \mathrm{PIB} *$} \\
\cline { 2 - 7 } & tendencia & intercepto & sin constante & tendencia & intercepto & sin constante \\
\hline Argentina & -1.3 & 0.994 & 2.575 & -1.197 & 0.448 & 1.298 \\
Bolivia & -1.022 & -0.675 & 0.728 & -2.137 & $-2.062^{* *}$ & 0.774 \\
Brasil & -1.171 & 1.599 & 2.777 & -2.993 & -1.752 & 0.246 \\
Chile & -0.97 & 1.776 & 3.656 & -2.405 & -0.306 & 1.531 \\
Colombia & -1.549 & 0.349 & 1.406 & $-3.528^{*}$ & -1.053 & 0.407 \\
Costa Rica & -1.139 & 0.953 & 3.412 & -2.635 & -1.304 & 0.819 \\
Ecuador & -1.833 & -0.143 & 1.898 & -2.877 & -2.052 & 0.325 \\
El Salvador & -0.391 & 1.189 & 2.433 & -1.604 & -1.526 & 0.558 \\
Guatemala & -1.375 & -1.361 & -0.022 & -1.59 & -0.874 & 0.975 \\
Honduras & -2.424 & -1.88 & 0.374 & -0.885 & 0.494 & 2.118 \\
Jamaica & -1.939 & -1.35 & 0.28 & $-3.633 * *$ & -2.483 & -0.014 \\
México & -0.217 & 3.422 & 5.318 & -2.229 & -0.214 & 1.195 \\
Nicaragua & -1.288 & 0.35 & 2.045 & -3.06 & $-2.735^{* *}$ & -0.045 \\
\hline
\end{tabular}




\begin{tabular}{|l|l|c|c|l|l|c|}
\hline & \multicolumn{3}{|c|}{ (X+M)/PIB } & \multicolumn{3}{c|}{$(\mathrm{X}+\mathrm{M}) / \mathrm{PIB} *$} \\
\cline { 2 - 7 } & tendencia & intercepto & sin constante & tendencia & intercepto & sin constante \\
\hline Panamá & $-3.318^{* * *}$ & $-2.71^{*}$ & -0.43 & -2.204 & $-2.228^{* *}$ & -0.218 \\
Paraguay & -1.829 & -0.876 & 0.353 & -2.423 & -0.551 & 0.684 \\
Perú & -0.252 & 0.236 & 1.1 & -3.274 & $-3.386^{*}$ & -0.381 \\
Rep. Dominicana & -2.582 & $-2.2 * *$ & -0.622 & -2.694 & -1.619 & -0.032 \\
Trinidad y Tobago & -2.479 & -0.244 & 1.604 & -1.922 & -2.238 & -0.581 \\
Uruguay & -2.096 & 0.73 & 2.779 & -2.61 & -1.178 & 0.485 \\
Venezuela & $-3.085^{*}$ & -3.101 & -0.083 & -3.119 & $-2.461^{* *}$ & 0.153 \\
\hline
\end{tabular}

$* * *, * *, *$ significativos al $10 \%, 5 \%$ y $1 \%$, respectivamente.

\begin{tabular}{|l|l|l|l|l|l|c|}
\hline & \multicolumn{3}{|c|}{$1-\mathrm{r}$} & \multicolumn{3}{c|}{$\mathrm{u}$} \\
\cline { 2 - 7 } & tendencia & intercepto & sin constante & tendencia & intercepto & sin constante \\
\hline Argentina & -2.438 & -1.989 & 0.15 & -1.382 & 0.655 & -1.602 \\
Bolivia & -2.702 & -1.673 & 1.128 & -1.346 & -1.25 & -1.059 \\
Brasil & -2.253 & -2.274 & -2.152 & -1.568 & 0.969 & 3.831 \\
Chile & $-8.528 *$ & $-8.072 *$ & 1.589 & -0.974 & 1.785 & 1.482 \\
Colombia & -2.586 & -0.636 & 1.005 & -2.946 & -1.431 & -1.216 \\
Costa Rica & -2.027 & -1.284 & 0.265 & -1.164 & 0.909 & 1.765 \\
Ecuador & -2.434 & -2.527 & 0.352 & -2.433 & -0.494 & -0.917 \\
El Salvador & -2.543 & -0.378 & 0.772 & -0.363 & 1.09 & -0.302 \\
Guatemala & -2.364 & -1.243 & 0.364 & -1.439 & -1.46 & -1.514 \\
Honduras & -1.708 & 0.066 & 1.1 & -2.426 & -2.104 & 0.307 \\
Jamaica & $-6.83^{* *}$ & $-4.579 * *$ & 1.408 & -1.68 & -1.424 & -1.602 \\
México & -3.044 & -2.021 & 1.14 & -0.296 & 2.385 & 0.243 \\
Nicaragua & $-5.188^{* *}$ & $-4.926 *$ & -0.176 & -0.99 & 0.34 & -1.242 \\
Panamá & -3.324 & $-2.738^{*}$ & 0.623 & -3.156 & $-2.964 *$ & -1.049 \\
Paraguay & -1.538 & -2.148 & 1.882 & -1.79 & -0.837 & -0.442 \\
Perú & $-3.744^{* *}$ & -1.68 & 0.505 & -0.184 & 0.416 & -1.243 \\
Rep. Dominicana & -1.908 & -1.98 & 0.991 & -2.675 & $-2.696 *$ & -0.503 \\
Trinidad y Tobago & $-6.112^{*}$ & -0.783 & 0.251 & -2.369 & -0.087 & 0.939 \\
Uruguay & $-5.065^{*}$ & -2.731 & 0.112 & -2.176 & 0.595 & -1.973 \\
Venezuela & -2.79 & $-2.579 * *$ & 0.017 & -2.964 & $-3.004 *$ & -1.981 \\
\hline
\end{tabular}

$* * *, * *, *$ significativos al $10 \%, 5 \%$ y $1 \%$, respectivamente. 
III. Gráficos de las series de PTF y apertura por país para el período 19602005
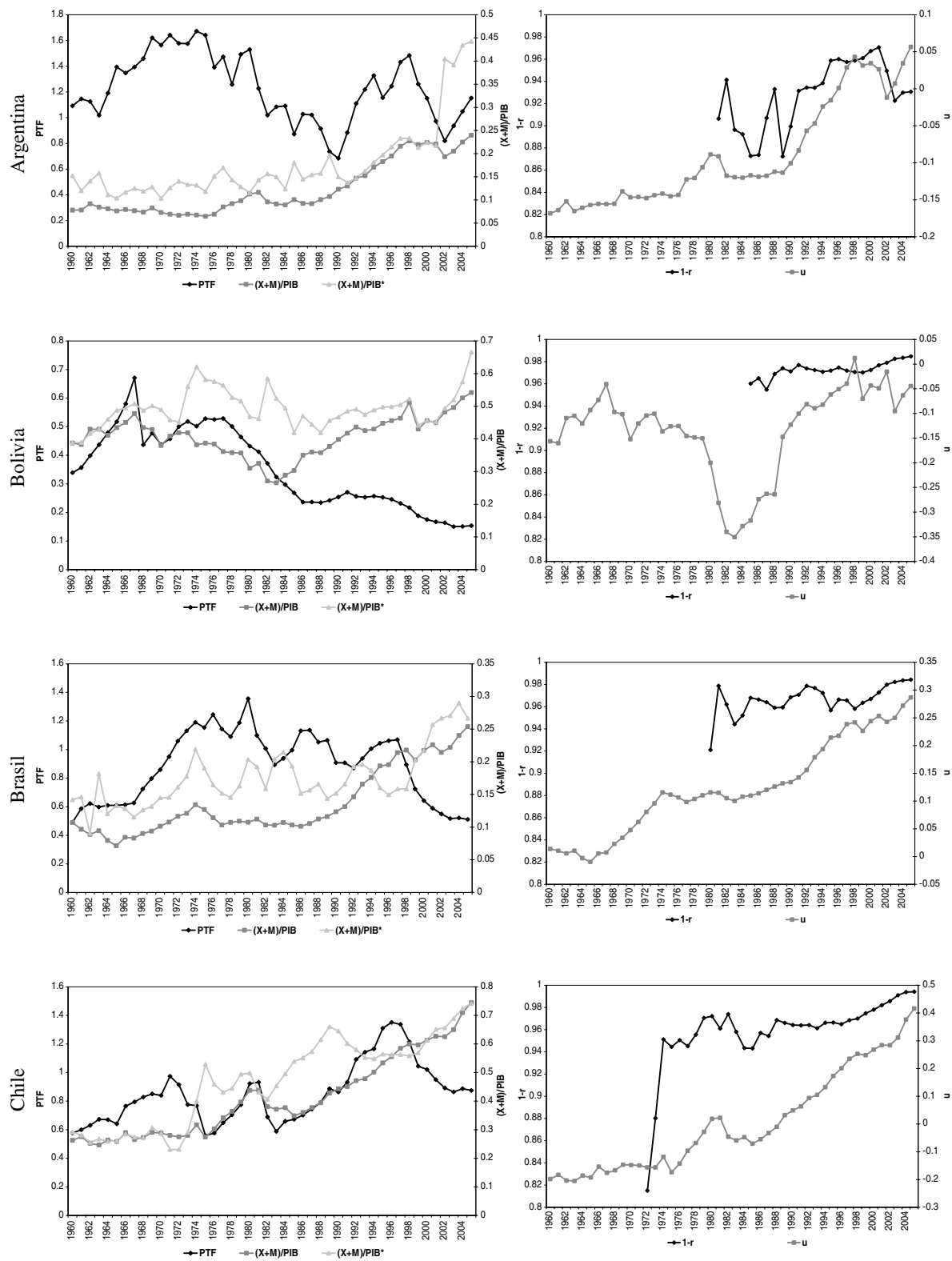

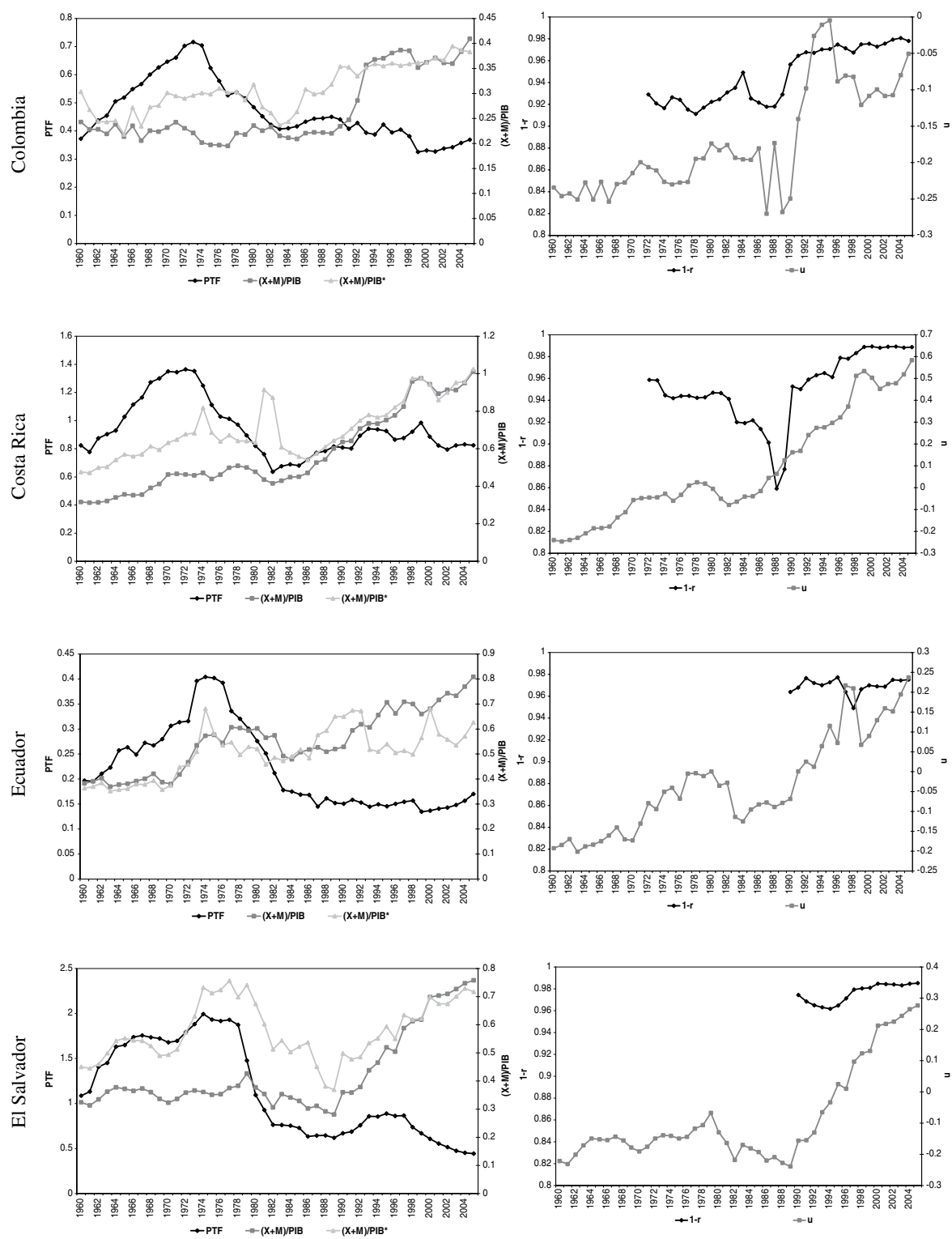

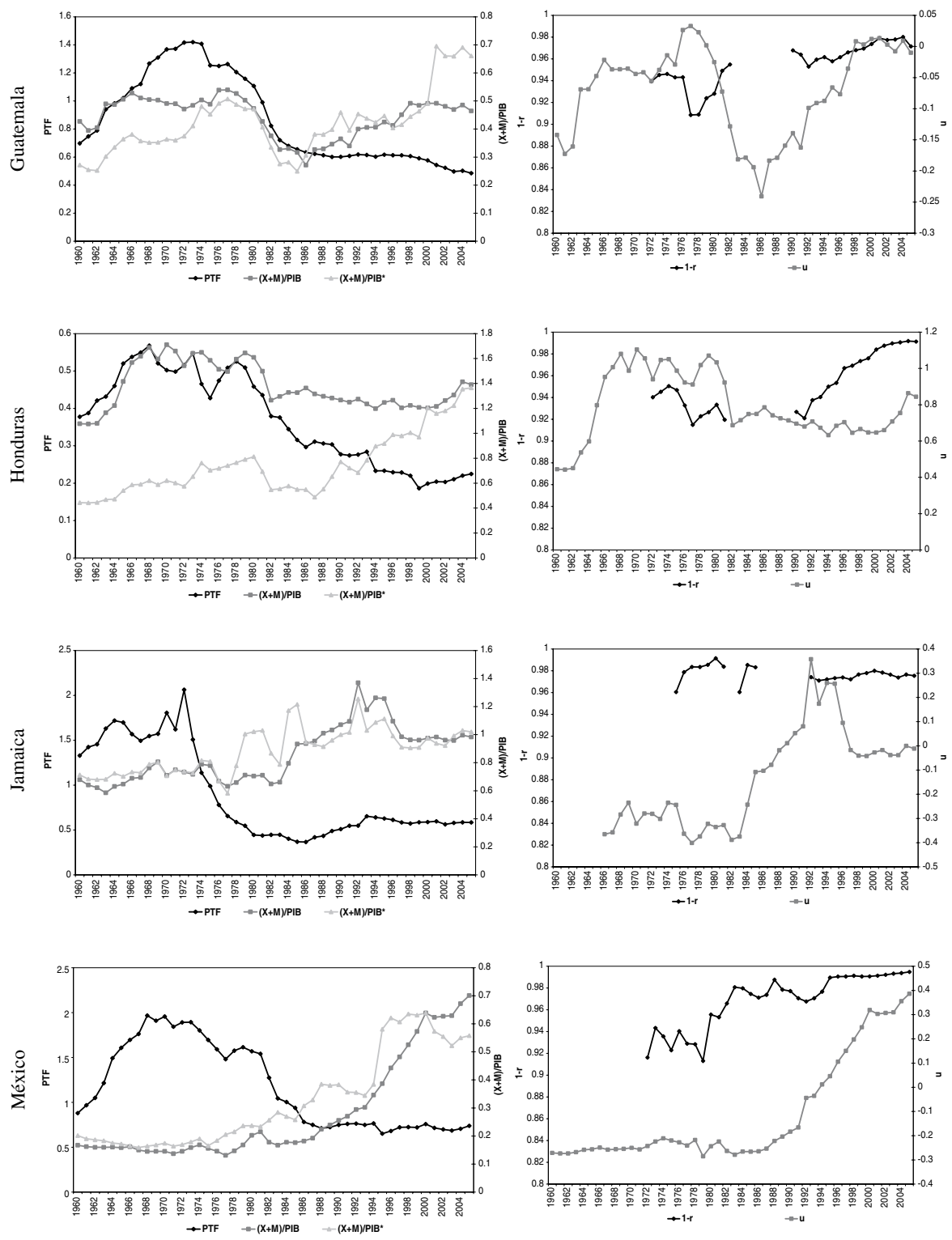


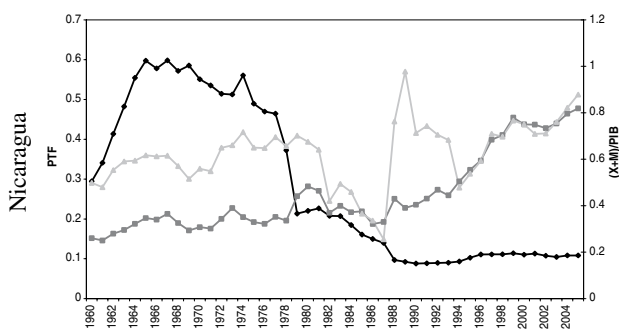

$\rightarrow$ PTF $\quad-(X+M) / P I B \quad \rightarrow(X+M) / P^{\prime B} B^{*}$
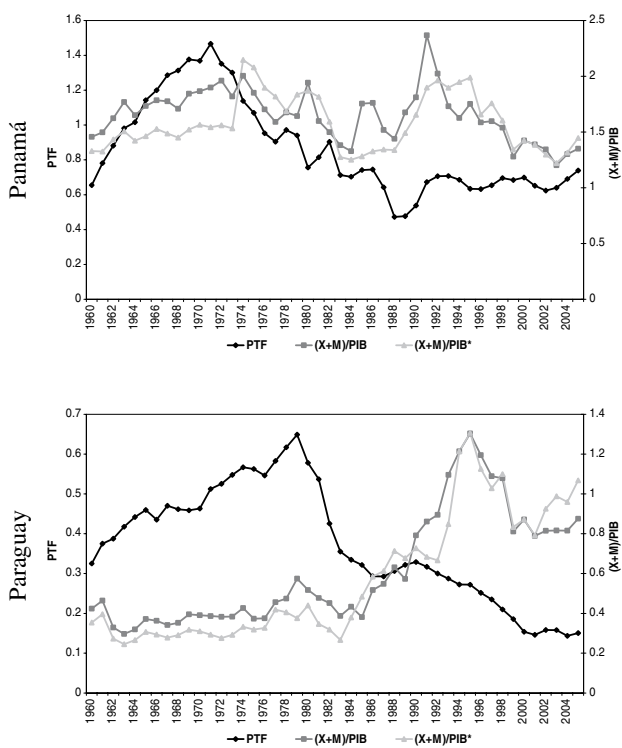

$\rightarrow$ PTF $\rightarrow(X+M) / P I B \quad-(X+M) / P I B *$

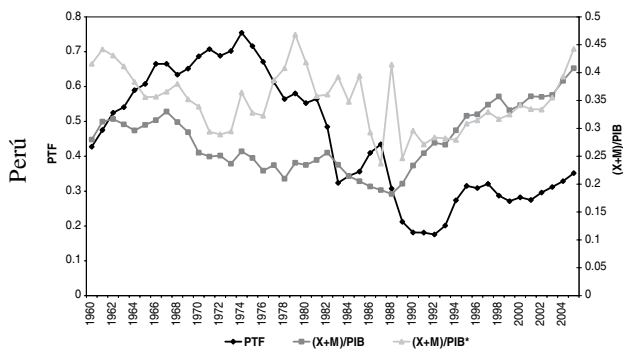

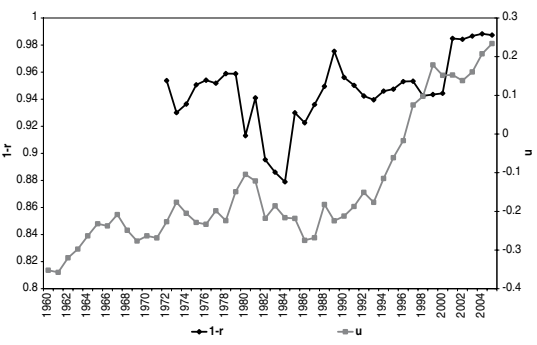
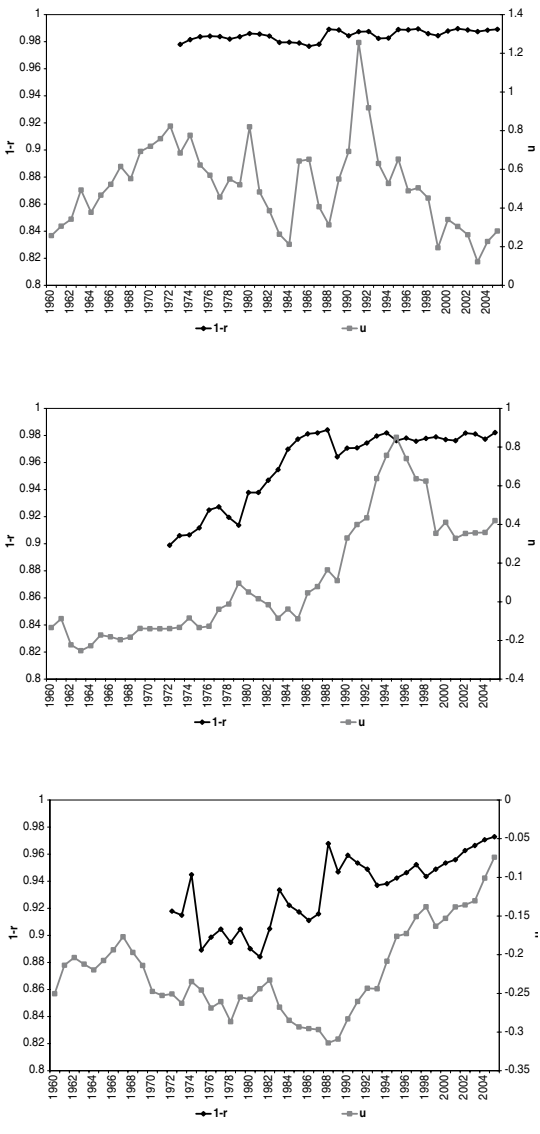

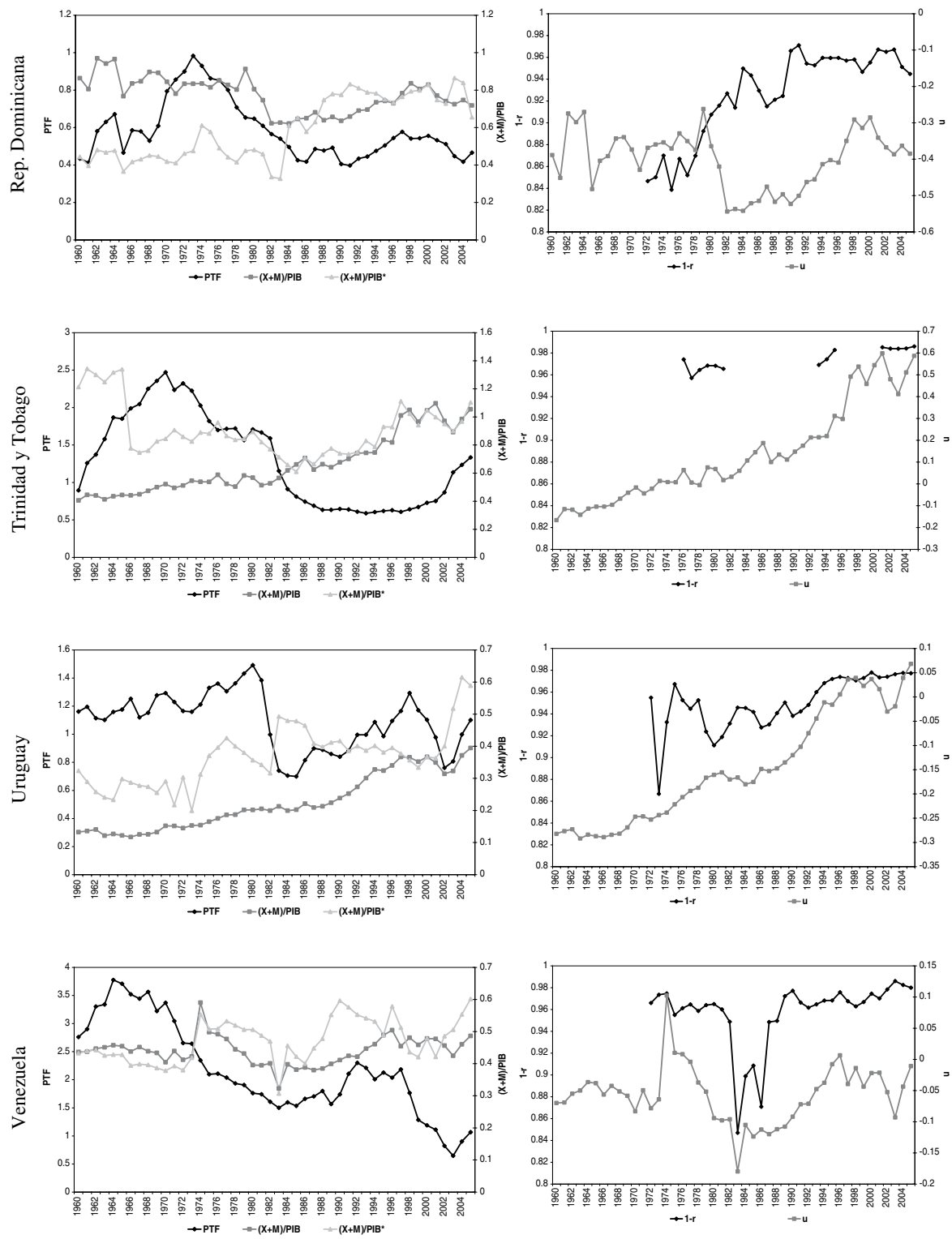
